\title{
Research on Risk Assessment of Smart Grid Project
}

\author{
Wanyu Xu \\ Department of Electrical Engineering,North China Electric Power University,Baoding, China \\ 757968298@qq.com
}

Keywords: Smart grid; Risk assessment; Rank correlation analysis; Gray triangle clustering Abstract. In light of the current development of smart grid in China, this paper established the risk assessment index system, and conducted a comprehensive evaluation based on the rank correlation analysis and gray triangle clustering method. Calculation examples indicate that the risk level of our smart grid project is between medium and poor, approximately consistent with the present technical development and social status in China.

\section{Introduction}

The research on the smart grid at home and abroad are in the initial stage, and the smart grid project risk assessment is also less[1]. Based on the current development of the smart grid, this paper establishes project risk assessment index system, uses the rank correlation analysis to calculate the relationship between the assessment index weight coefficient, and uses gray triangle clustering method to make a comprehensive risk assessment for smart grid project.

\section{Risk assessment index system of smart grid project}

Establish the assessment index system.

After the introduction of the smart grid, the risk caused by the failure of original equipment still exists. And the structural changes brought by the smart grid will make risk analysis becomes more complex[2]. It is necessary to reduce and control the risk from the controllable factors. According to the current development of the smart grid, this paper divide the smart grid project risk into five categories:

(1) Project risk, which includes the address selection (V1), project scale level (V2), the project investment risk (V3) as well as political, economic and legal policy risks (V4).

(2) Technical risk, which is the main risk of smart grid construction, mainly includes the core technology maturity (V5), technical implementation (V6) and technical changes (V7).

(3) Environmental risks. Building smart grid will improve the ability to accept renewable energy sources, but it will make some negative influences to the human living environment, mainly includes geological disasters (V8), destruction of hydrological conditions (V9) and environmental impact assessment pass rate(V10) and so on.

(4) Security risk, which is the primary requirement of smart grid, mainly includes system stability (V11), power supply reliability (V12), number of equipment accidents (V13) and information security level (V14).

(5) Management risk, which means the risks caused by the misjudgment and information asymmetry in the process of power grid operation, mainly includes funding adequacy (V15), technical personnel quality (V16) and implement degree of the progress (V17) .

For the properties of assessment object, set $m$ assessment indicators and $s$ different gray level. The sample prediction value of assessment object $i$ to indicator $j$ is defined as $X_{i j}$, 
$j=1,2, \cdots, m$. According to the value of $X_{i j}$, make an assessment to object $i[3]$.

\section{Determine the indicator weight.}

The rank correlation analysis is a subjective weighting method, and the core idea is that the experts take the relative importance sort of influencing factors according to the assessment criteria and determine the relative importance value of the impact of adjacent indicators. Then, compare the importance degree of resort indicators and give the ratio. Finally, the weight coefficients of indicators are obtained. The processes are as follows[4]:

(1) Determine the rank correlation

The experts select the most important indicator from index set $\left\{t_{1}, t_{2}, \cdots, t_{n}\right\}$, and denote it as $t_{1}^{*}$. Then, the experts select the most important indicator from the remaining $n-1$ indicators and denote it as $t_{2}^{*}$. Repeat the above steps. After $n-1$ selection, the last remaining indicator is denoted as $t_{n}^{*}$. Thus, the new rank correlation among indicators is obtained: $t_{1}^{*}>t_{2}^{*}>\cdots>t_{n}^{*}$.

(2) Calculate the comparison value between $t_{k-1}^{*}$ and $t_{k}^{*}$

The importance comparison ratio between indicator $t_{k-1}^{*}$ and indicator $t_{k}^{*}$ is shown as follows:

$$
\varphi_{k}=\frac{\beta_{i-1}^{*}}{\beta_{i}^{*}}, k=n, n-1, \cdots, 2
$$

When $n$ is large, the value of $\varphi_{k}$ can choose 1 according to rank correlation $t_{1}^{*}>t_{2}^{*}>\cdots>t_{n}^{*}$. The assignment of $\varphi_{k}$ can refer Table. 1 .

Table. 1 The reference of $\varphi_{k}$ assignment

\begin{tabular}{|c|c|}
\hline$\varphi_{k}$ & 说明 \\
\hline 1.0 & $t_{k-1}^{*}$ is as important as $t_{k}^{*}$ \\
\hline 1.2 & $t_{k-1}^{*}$ is somewhat important than $t_{k}^{*}$ \\
\hline 1.4 & $t_{k-1}^{*}$ is obviously important than $t_{k}^{*}$ \\
\hline 1.6 & $t_{k-1}^{*}$ is highly important than $t_{k}^{*}$ \\
\hline 1.8 & $t_{k-1}^{*}$ is extremely important than $t_{k}^{*}$ \\
\hline
\end{tabular}

(3) Calculate the weight value

According to the comparison judgment value, we can require the following weight calculation equation.

$$
\begin{aligned}
& \bar{x}_{n}=\left(1+\sum_{k=2}^{n} \prod_{i=k}^{n} \varphi_{i}\right)^{-1} \\
& \bar{x}_{k-1}=\varphi_{k} * \bar{x}_{k}, \quad k=n-1, n-2, \cdots, 2
\end{aligned}
$$

\section{Gray triangle clustering assessment model}

(1) The number of gray level is determined as $m$ according to assessment requirement. Divide the indicator range into $m$ gray levels. For example, divide the range of indicator $j\left[p_{1}, p_{m+1}\right]$ into $m$ intervals: $\left[p_{1}, p_{2}\right],\left[p_{2}, p_{3}\right], \cdots,\left[p_{m}, p_{m+1}\right]$.

(2) Set $\gamma_{k}=\left(p_{k}+p_{k+1}\right) / 2$, the value of gray triangle clustering which belongs to $k$ gray level is 1. Connect $\left(\gamma_{k}, 1\right)$ with the start point $p_{k+1}$ of $k-1$ gray level and the end point $p_{k+2}$ of 
$k+1$ gray level. Then, the gray triangle clustering function $\varepsilon_{k j}($.$) which belongs to k$ gray level of indicator $j$ is obtained, and $j=1,2, \cdots, m, k=1,2, \cdots, s$. For $\varepsilon_{j 1(\cdot)}$ and $\varepsilon_{j s}(\cdot)$, it can take the range of indicator $j$ left to $J_{0}$ and right to $J_{s+2}$. Thus, the gray triangle clustering function of indicator $j$ is shown as follow.

$$
\varepsilon_{j}^{k}(l)= \begin{cases}0, \quad l \in\left[p_{k-1}, p_{k+2}\right] \\ \frac{l-p_{k-1}}{\gamma_{k}-p_{k-1}}, \quad l \in\left[p_{k-1}, \gamma_{k}\right] \\ \frac{p_{k+2}-l}{p_{k+2}-\gamma_{k}}, & l \in\left[\gamma_{k}, p_{k+2}\right]\end{cases}
$$

If the indicator $j$ 's prediction value of object $i$ is 1 , the membership $\varepsilon_{j k}(l)$ of gray level $k$ can be calculated due to Eq. (1).

(3) Calculate the gray assessment coefficient and its weight vector. For assessment indicator $H_{i}$, the gray assessment belonging to $e$ gray level is denoted as $q_{i \varepsilon}$. The number of total assessment belonging to different gray levels is denoted as $q_{i}$. Thus,

$$
\begin{aligned}
& q_{i \varepsilon}=\sum_{j=1}^{m} f_{\varepsilon}\left(d_{i j}\right), \quad i=1,2, \cdots, n \\
& q_{i}=\sum_{\varepsilon=1}^{4} q_{i j}, \quad i=1,2, \cdots, n
\end{aligned}
$$

According to Eq. (5) and Eq. (6), the gray assessment weight of $e$ gray level belonging to indicator $B_{i}$ is $\gamma_{i \varepsilon}=q_{i \varepsilon} / q_{i}$. And each assessment indicator's gray assessment weight vector is $\gamma_{i}=\left(\gamma_{i 1}, \gamma_{i 2}, \gamma_{i 3}, \gamma_{i 4}\right)$.

(4) For the comprehensive assessment of index layer $B_{i}$, its assessment result is denoted as $H_{i}$. Thus,

$$
H_{i}=B_{i} * U_{i}=\left(h_{i 1}, h_{i 2}, h_{i 3}, h_{i 4}\right), \quad i=1,2,3
$$

The above assessment results construct the principle layer $J$, and the gray weight matrix of each assessment gray level is obtained.

$$
H=\left[\begin{array}{l}
H_{1} \\
H_{2} \\
H_{3}
\end{array}\right]=\left[\begin{array}{llll}
h_{11} & h_{12} & h_{13} & h_{14} \\
h_{21} & h_{22} & h_{23} & h_{24} \\
h_{31} & h_{32} & h_{33} & h_{34}
\end{array}\right]
$$

For the comprehensive assessment of principle layer, its assessment result is as follows:

$$
U=J H=\left(U_{1}, U_{2}, U_{3}, U_{4}\right)
$$

For the comprehensive assessment results $U$, according to the maximum principle, calculate the comprehensive assessment value $F=U * B^{T}$, where $B$ is the assignment vector according to gray level. This paper divides the comprehensive assessment value into four gray levels.

\section{Case study}

\section{Determine indicators' weight.}

(1) Determine rank correlation

This paper invited experts to sort the rank correlation, through comparing the relative 
importance of the listed indicators, the priority rank correlation is obtained.

$\mathrm{V} 5>\mathrm{V} 4>\mathrm{V} 12>\mathrm{V} 15>\mathrm{V} 3>\mathrm{V} 17>\mathrm{V} 11>\mathrm{V} 6>\mathrm{V} 13>\mathrm{V} 2>\mathrm{V} 7>\mathrm{V} 1>\mathrm{V} 16>\mathrm{V} 10>\mathrm{V} 14>\mathrm{V} 8>\mathrm{V} 9$

(2) Obtain the judgment comparison value

According to Eq. (1), the comparison value $\sigma$ from experts' judgment, which is shown in Table. 2.

Table. 2 Experts' judgment comparison value

\begin{tabular}{|c|c|c|c|c|c|c|c|}
\hline$\sigma_{2}$ & $\sigma_{3}$ & $\sigma_{4}$ & $\sigma_{5}$ & $\sigma_{6}$ & $\sigma_{7}$ & $\sigma_{8}$ & $\sigma_{9}$ \\
\hline 1 & 1.8 & 1.2 & 1.6 & 1.6 & 1.6 & 1.2 & 1.6 \\
\hline$\sigma_{10}$ & $\sigma_{11}$ & $\sigma_{12}$ & $\sigma_{13}$ & $\sigma_{14}$ & $\sigma_{15}$ & $\sigma_{16}$ & $\sigma_{17}$ \\
\hline 1.4 & 1.4 & 1 & 1.6 & 1.2 & 1.2 & 1.6 & 1.4 \\
\hline
\end{tabular}

(3) Calculate weight coefficient

According to the judgment comparison value in Table. 2, based on Eq. (2) and Eq. (3), calculate the weight of indicators, and the results are shown in Table. 3.

Gray triangle clustering assessment model.

(1) This paper divides the gray level into for levels including excellent, good, medium and poor. Apply 10-point scale assessment criteria and set the range between 0 and 10. The intervals corresponding to four levels are respectively $[0,2.5],[2.5,5],[5,7.5],[7.5,10]$.

(2) The basic data values are obtained from the long term experience of experts which is shown in Table. 3.

Table. 3 The weight and basic data values of indicators

\begin{tabular}{|c|c|c|}
\hline Indicators & Weight & Basic value \\
\hline V1 & 0.009 & 6.2 \\
\hline V2 & 0.013 & 8,3 \\
\hline V3 & 0.091 & 5.5 \\
\hline V4 & 0.237 & 8,6 \\
\hline V5 & 0.237 & 6.4 \\
\hline V6 & 0.03 & 5.7 \\
\hline V7 & 0.009 & 7.7 \\
\hline V8 & 0.003 & 3.4 \\
\hline V9 & 0.002 & 4.2 \\
\hline V10 & 0.005 & 8.9 \\
\hline V11 & 0.036 & 8.8 \\
\hline V12 & 0.132 & 9.3 \\
\hline V13 & 0019 & 2.4 \\
\hline V14 & 0.004 & 8.9 \\
\hline V15 & 0.110 & 7.8 \\
\hline V16 & 0.006 & 7.4 \\
\hline V17 & 0.057 & 8.9 \\
\hline
\end{tabular}

(3) According to Eq. (4) and Table. 3, calculate the membership of assessment indicators which are shown in Table. 4.

(4) According to Eq. (6), require the gray clustering weight vectors of indicator which are shown in Table. 4. 
Table. 4 The membership and gray clustering weight vector of indicators

\begin{tabular}{|c|c|c|c|c|c|c|c|c|}
\hline Indicator & \multicolumn{5}{|c|}{ Membership } & \multicolumn{4}{c|}{ Gray clustering weight vector } \\
\hline V1 & 0 & 0.35 & 0.99 & 0.32 & 0 & 0.21 & 0.60 & 0.19 \\
\hline V2 & 0 & 0 & 0.45 & 0.88 & 0 & 0 & 0.34 & 0.66 \\
\hline V3 & 0 & 0.53 & 0.80 & 0.13 & 0 & 0.36 & 0.55 & 0.09 \\
\hline V4 & 0 & 0 & 0.37 & 0.96 & 0 & 0 & 0.28 & 0.72 \\
\hline V5 & 0 & 0.29 & 0.96 & 0.37 & 0 & 0.18 & 0.59 & 0.23 \\
\hline V6 & 0 & 0.48 & 0.85 & 0.19 & 0 & 0.32 & 0.56 & 0.12 \\
\hline V7 & 0 & 0 & 0.61 & 0.72 & 0 & 0 & 0.46 & 0.54 \\
\hline V8 & 0.43 & 0.91 & 0.24 & 0 & 0.27 & 0.58 & 0.15 & 0 \\
\hline V9 & 0.21 & 0.88 & 0.45 & 0 & 0.14 & 0.57 & 0.29 & 0 \\
\hline V10 & 0 & 0 & 0.29 & 0.96 & 0 & 0 & 0.23 & 0.77 \\
\hline V11 & 0 & 0 & 0.32 & 0.99 & 0 & 0 & 0.24 & 0.76 \\
\hline V12 & 0 & 0 & 0.19 & 0.85 & 0 & 0 & 0.14 & 0.65 \\
\hline V13 & 0.69 & 0.64 & 0 & 0 & 0.53 & 0.49 & 0 & 0 \\
\hline V14 & 0 & 0 & 0.29 & 0.96 & 0 & 0 & 0.22 & 0.73 \\
\hline V15 & 0 & 0 & 0.59 & 0.75 & 0 & 0 & 0.45 & 0.57 \\
\hline V16 & 0 & 0.03 & 0.69 & 0.64 & 0 & 0.02 & 0.53 & 0.49 \\
\hline V17 & 0 & 0 & 0.29 & 0.96 & 0 & 0 & 0.22 & 0.73 \\
\hline
\end{tabular}

(5) Make comprehensive assessment for index layer $Y_{i}$, denote its result as $T_{i}$. Thus, $T_{i}=Y_{i} * U_{i}=\left(T_{i 1}, T_{i 2}, T_{i 3}, T_{i 4}\right), \quad i=1,2, \cdots, 17$

For example, $\quad T_{1}=\left\{\begin{array}{llll}0 & 0.099 & 0.359 & 0.541\end{array}\right\} \quad, \quad T_{2}=\left\{\begin{array}{lllll}0 & 0.189 & 0.583 & 0.228\end{array}\right\} \quad$ and $T=\left[T_{1}, \cdots, T_{17}\right]^{T}$.

Make comprehensive assessment for principle layer $K$, denote its result as $A$ : $A=J T=\left(\begin{array}{llll}0.011 & 0.099 & 0.383 & 0.480\end{array}\right)$. According to the maximum principle, the comprehensive assessment value is calculated as 4.151 , which indicates that the risk level is between medium level and poor level.

\section{Conclusions}

Through the establishment of a smart grid project risk assessment model, in the project process, taking into consideration all aspects of stage before and after the construction process is conducive to fully understand and assess a variety of risks facing the construction of smart grid. The numerical example analysis indicates that the risk level of our smart grid project is medium level and poor level, which meets China's current economic situation and the level of technology.

\section{References}

[1] ZENG Ming, CHEN Ying-jie, HU Xian-zhong, et al. The risk assessment of China' s smart grid based on multi-level fuzzy comprehensive evaluation method. East China Electric Power. Vol. 39 (2011), p. 535-539.

[2] ZHAO Shan-shan, ZHANG Dong-xia, YIN Yong-hua, et al. Risk assessment of smart grid. Power System Technology. Vol. 33 (2009), p. 7-10.

[3] HOU Li-li, XU Zhi-fan. Security evaluation of smart grid based on AHP-Entropy method. Modern Electric Power. Vol. 28 (2011), p. 85-89.

[4] DIXON R K, McGOWAN E, ONYSKO G.US energy conservation and efficiency policies: Challenges and opportunities. Energy Policy. Vol. 38 (2010), p. 6398-6408. 